\title{
Oligometastatic Disease in Pancreatic Cancer - How to Proceed?
}

\author{
Bernhard W. Renz $z^{a, b}$ \\ Volker Heinemann $n^{b, c}$ \\ Stefan Boeck $k^{b, c}$ \\ Falk Roeder ${ }^{b, d, e} \quad$ Christoph Trumm ${ }^{f}$
}

a Department of General, Visceral and Transplantation Surgery, Hospital of the University of Munich, Munich, Germany;

${ }^{b}$ Pancreatic Cancer Center Munich, Comprehensive Cancer Center-LMU, University of Munich, Munich, Germany;

${ }^{c}$ Department of Internal Medicine III, Hospital of the University of Munich, Munich, Germany;

${ }^{\mathrm{d}}$ Department of Radiation Oncology, Hospital of the University of Munich, Munich, Germany;

e Department of Molecular Radiation Oncology, German Cancer Research Center, Heidelberg, Germany;

${ }^{f}$ Department of Clinical Radiology, Hospital of the University of Munich, Munich, Germany

\section{Keywords}

Pancreatic cancer - Oligometastatic pancreatic ductal adenocarcinoma $\cdot$ Isolated metastasis

\section{Summary}

Background: Pancreatic cancer is a highly aggressive malignancy and will become the second leading cause of cancer-related death in the USA and also in Germany by 2030 . Furthermore, the majority of patients with pancreatic ductal adenocarcinoma (PDAC) will present with distant metastases, limiting surgical management in this population as there is little evidence available to support surgical or ablative treatment options for advancedstage disease. However, highly selected patients suffering from synchronous and metachronous oligometastatic PDAC may potentially benefit from a surgical resection with an acceptable morbidity. Methods: This review summarizes and discusses the current literature on the management of oligometastatic disease regarding PDAC, focusing on para-aortic lymph nodes as well as isolated hepatic and pulmonary metastases. Results and Conclusion: In order to further investigate the feasibility and efficacy of such an approach, a prospective multicenter trial, in which survival and quality of life after metastatic resection and systemic chemotherapy is evaluated, has to be initiated. Additionally, local and locoregional ablation techniques or stereotactic body radiation therapy as therapeutic options for isolated metastases in PDAC need further research in order to determine their significance and benefit.

(C) 2017 S. Karger GmbH, Freiburg

\section{Introduction}

Metastatic pancreatic ductal adenocarcinoma (PDAC) has a dismal prognosis with a median overall survival of about 6 months and will become the second leading cause of cancer-related death in the USA and also in Germany by $2030[1,2]$. It is therefore one of the most lethal cancers, as indicated by a very high mortality-toincidence ratio [3]. In contrast to the steady increase in survival for most cancers, advances have been slow for pancreatic cancer, for which the 5 -year relative survival rate is currently $8 \%$. These low rates are explained by the fact that more than $50 \%$ of cases are diagnosed at a distant stage discovered by imaging at the time of diagnosis or during attempted pancreatic resection. Furthermore, another $30 \%$ present with locally advanced pancreatic cancer $[3,4]$.

Pancreatic cancer metastasizes primarily to the liver, peritoneum, and lungs [5]. However, distant metastases of PDAC have been reported in almost every organ, including bones and adrenal glands [6-9], the brain and leptomeninges, diaphragm, gallbladder, heart and pericardium, small and large intestines, kidneys, ovaries and uterus, seminal vesicles, skin, stomach, spleen, testis, thyroid gland, urinary bladder, as well as orbit [7, 9-19].

It is widely accepted that surgical resection remains a vital necessity for a potential cure of this cancer entity. Over the past decades, the median overall survival has increased to 25-30 months after surgical resection in combination with adjuvant chemotherapy, and 5-year survival is well over $20 \%$ in these patients but still remains poor due to the high propensity of the tumor for locoregional and systemic recurrence $[4,20,21]$. In such palliative settings, therapeutic regimes, such as FOLFIRINOX or gemcitabine and nab-paclitaxel, have been established as the standard of care very recently $[22,23]$. Although palliative chemotherapy is the

\section{KARGER}

(๑) 2017 S. Karger GmbH, Freiburg

Fax +497614520714
Prof. Dr. Jens Werner, MBA

Klinik für Allgemein-, Viszeral- und Transplantationschirurgie

Klinikum der Universität München, LMU

Marchioninistraße 15, 81377 München, Germany

Jens.Werner@med.uni-muenchen.de 
standard of care for patients with metastatic disease [24], management of the subgroup of patients with oligometastatic disease is not clear. Therefore, unlike in other malignancies, synchronous or metachronous metastasectomy of PDAC is rarely performed in current clinical practice.

Several treatment options, including neoadjuvant therapy with subsequent resection as well as ablative technologies, should be considered. However, there is little evidence available to support treatment options for oligometastatic disease. As valid predictive biomarkers for stratification of therapy are not available today, future trials need to define the role of the different treatment options. This review summarizes the current evidence and discusses available treatment options for oligometastatic PDAC with a specific focus on para-aortic lymph nodes (PALN) as well as on metastases to the liver and lungs.

\section{Para-Aortic Lymph Nodes}

PALN (Group 16 according to the Japanese Pancreas Society) [25] are considered as 'extra-regional' lymph nodes and are involved only after the metastatic spread has already reached the peri-pancreatic first-echelon lymph nodes [26]. Although positive nodes in this group are considered as distant metastases (M1 disease), their prognostic value remains controversial [27]. Recently, a systematic review by the Bassi group was performed [26]. Here, 13 studies were included and PALN metastasis appeared to correlate with poor prognosis in patients with PDAC. The largest study was a retrospective multicenter analysis of 882 patients who had undergone pancreatic resection with pathological evaluation of PALN for PDAC [28]. Patients with metastatic PALN in this study had a significantly poorer median survival than those without (17 vs. 23 months; $\mathrm{p}=0.001$ ). In contrast, Shrikhande et al. [29] compared outcome after resection for M1 pancreatic cancer, including a subgroup with positive PALN. In this study, survival of these patients was significantly better ( 27 months) than in the subgroup with resected liver (11.4 months) or peritoneal metastases (12.9 months) and was comparable to node-negative patients. In contrast, in a larger study from the University of Heidelberg, a median survival of 12.3 months was reported in $n=43$ patients with resected positive PALN [30]. Therefore, as the data remains inconsistent, it seems difficult to draw a final conclusion concerning this issue, which is also reflected in a recent consensus statement by the International Study Group on Pancreatic Cancer [25].

\section{Hepatic Metastasis}

\section{Role of Surgery}

More than $90 \%$ of patients who are diagnosed with PDAC die from the disease. Approximately $70 \%$ of these patients have extensive metastatic disease at the time of death, with $30 \%$ having limited metastatic disease, but many of them have bulky primary tumors [29]. As already mentioned, the liver is the most common site of PDAC metastasis.
For colorectal liver metastases, despite the availability of alternative therapeutic options, hepatectomy remains the treatment of choice. It has been proven to be safe and oncologically beneficial, extending survival and improving quality of life [31,32]. After curative resection of colorectal liver metastases, 5-year survival rates range from 28 to $60 \%$ depending on strategies and preoperative selection criteria [33]. Likewise, resection for hepatic metastases of neuroendocrine tumors, including pancreatic neuroendocrine tumors, is widely accepted with the objective of symptom control and improved longterm outcome [34]. However, in patients undergoing liver resection for non-colorectal, non-neuroendocrine liver metastases, patient selection seems to be even more critical than in colorectal liver metastasis or primary liver tumors [35]; however, the significance of surgery has not been satisfactorily elucidated, especially regarding longterm outcome. Thus, there are still controversies concerning the oncological value of liver surgery in these patients. Therefore, even in high-volume centers synchronous liver and pancreatic resections are performed in very few PDAC cases [30].

Current national and international guidelines [36, 37] do not recommend resection of the primary tumor and synchronous liver metastases; as a result, this particular treatment is only being performed in highly selected patients. It has to be noticed that in most cases the decision for an intentional resection in a patient with PDAC metastasized to the liver is made after subjective considerations of the surgeon. Ideally, this decision is based on a highly individual basis, including the patient's wishes, age, clinical status, local resectability, and the individual risk of complications. However, published data demonstrate that the procedure can be performed safely, but results are inconsistent as to whether complete resection of the PDAC with combined resection of liver metastases will lead to a survival benefit [30, 38-43]. Eight reports including more than 9 patients found median overall survival times between 5.9 and 11.4 months after resection [29, 39, 42, 44-48].

Klein et al. [44] reported a median survival in PDAC patients with hepatic metastases of 7.6 months after resection. Within this small study, 22 PDAC patients who underwent synchronous, liverdirected therapy either with anatomical liver resection (7 patients $(32 \%))$ or atypical resection (15 patients $(68 \%))$ were analyzed. All patients received adjuvant therapy with gemcitabine. Data to which extent the patients also received neoadjuvant treatment is not mentioned within the publication.

Gleisner et al. [42] reported that even among well-selected patients with low-volume metastatic liver disease, simultaneous resection of pancreatic carcinoma with synchronous liver metastasis did not result in long-term survival in the overwhelming majority of patients. In fact, the median survival of patients who underwent hepatic resection of synchronous metastasis was only 6 months, which was comparable to the survival of matched patients who underwent palliative bypass surgery only.

No benefit in overall survival in patients undergoing pancreatoduodenectomy with synchronous partial liver resection was also reported by Takada et al. [47].

Similar results were obtained by a study from Hanover. Here, a median survival of 8.3 months after synchronous liver and pancre- 
atic resection and 5.8 months after metachronous hepatic resection has been reported [40]. Even if the 1-year survival rates were $41 \%$ after synchronous resection and $40 \%$ after metachronous resection of hepatic metastases of pancreatic $(n=20)$ or ampullary $(n=2)$ cancers in this study, hepatic resection could not be recommended based on these data. More promising results were reported from Heidelberg. Here, 29 patients with metastatic PDAC who underwent synchronous metastasectomy were analyzed [29]. Out of these, 11 had hepatic resection for synchronous metastasis. These overall healthy patients (ASA > III) had only one or two isolated hepatic foci and a high probability that histologically negative resection margins could be achieved. Based on the significantly longer median overall survival of 11.4 months in the resected patients compared to 5.9 months in the group who underwent explorative laparotomy only, it was concluded that simultaneous liver resection for metastatic disease can be performed with acceptable safety in highly selected patients.

Most likely owing to a less selective cohort, the group from Mainz argued against simultaneous resection of the primary and liver metastases based on their experience [39]. In contrast, resection of metachronous PDAC liver metastases seems to improve survival in selected patients. The authors reported on 23 patients with metachronous and synchronous hepatic metastases. In 14 cases, liver metastases were found simultaneously, and in 9 cases metachronously. Of these, 13 patients underwent surgery and 10 were treated with gemcitabine only. There was no difference in survival in patients with synchronous liver metastases of PDAC treated by resection of the primary tumor combined with liver resection versus treatment by gemcitabine ( 8 vs. 11 months). In patients with metachronous liver metastases, the median survival was increased after metastasectomy compared to patients who were treated with gemcitabine only (31 vs. 11 months, respectively) [39].

Two studies including a larger number of patients were published very recently $[30,43]$. In a retrospective fashion, six European pancreas centers reported on 69 patients suffering from synchronous hepatic metastasized PDAC who underwent simultaneous pancreas and liver resections [43]. Patients who were explored, but in whom resection was not performed, served as controls. Data from this multicenter trial suggests a significant survival benefit with acceptable morbidity and mortality for patients receiving synchronous hepatic and pancreatic resection compared to patients with liver metastases who did not undergo surgery ( 14.5 vs. 7.5 months, respectively; $\mathrm{p}<0.001) .14 \%$ of the resected and $1 \%$ of the non-resected patients received neoadjuvant therapy $(\mathrm{p}=0.071)$. Similar results were reported in the single-center study from Heidelberg [30], in which $n=62$ patients underwent synchronous hepatic resection with a median survival of 10.6 months and $n=28$ patients had metachronous hepatic resection with a median survival of 14.8 months with acceptable morbidity and mortality. These results have to be compared to exclusive intensified chemotherapy regimen such as FOLFIRINOX with a median overall survival of 11.1 months [22]. It goes without saying that these studies also have many limitations, and general conclusions must be drawn cautiously. Though only PDAC patients were included, the analyzed groups are heterogeneous, and both PDAC of the head and the body/tail regions are analyzed.

As the FOLFIRINOX regimen is toxic and therefore more grade III and IV toxicities are encountered, this regimen may only be an appropriate option for a subset of patients. Until today we do not know whether patients who are not suitable for an intensified chemotherapy regimen according to their physical strength would do better after standard chemotherapy or synchronous resection regarding the assessment of quality of life.

This further highlights the need for assessments of quality of life when such palliative trials are performed. Given the fact that only a few patients in the study by Tachezy et al. [43] were treated with FOLFIRINOX as first- or second-line treatment due to the timeframe of the study, it is tempting to speculate that metastasectomized patients might benefit from a combination of both treatment approaches.

The question still remains:

- Which patients might benefit from such an individual approach? - Should only patients with stable disease or also progressive disease that appeared to regress after neoadjuvant therapy be offered aggressive combined resection, or should resection be performed in chemotherapy-naive patients with a small tumor burden?

Further research is needed to identify biomarkers for stratification of patients with low metastatic burden. In this regard, the predictive value of CA 19-9 was demonstrated in a retrospective cohort study [49]. It was suggested that CA 19-9 predicts resectability, stage of disease, as well as survival in PDAC patients. Highly elevated preoperative or increasing postoperative CA 19-9 levels were associated with low resectability and poor survival rates, and demanded the adjustment of surgical and perioperative therapy.

\section{Role of Ablation Techniques in Liver Metastasis}

Nowadays, it is not uncommon to utilize local percutaneous, locoregional transarterial as well as non-invasive local ablation techniques, including thermo-ablative approaches (radiofrequency ablation (RFA), microwave ablation, laser-induced thermal therapy, cryoablation, high-intensity focused ultrasound), chemo-ablative approaches (percutaneous ethanol injection, hepatic arterial infusion chemotherapy, transcatheter arterial chemoembolization and its variants), radio-ablative approaches (stereotactic body radiation therapy (SBRT), selective internal radiation therapy), and electroablative approaches (irreversible electroporation (Nanoknife ${ }^{\circledR}$ )) as tools of the multimodal treatment strategies of hepatic metastases from various kinds of tumors. During the last decade, the effectiveness and safety of these techniques have been shown for liver metastases [50-57]. These modalities are currently offered to selected patients with colorectal cancer liver metastasis but results vary due to tumor size, number, volume and location [58]. Accepted applications include inoperable disease due to tumor distribution or inadequate liver reserve. Furthermore, other current indications include concurrent comorbidity, patient choice, and the test-of-time approach. Future applications may include resectable disease, e.g. in patients with limited hepatic disease or with solitary liver metastasis $[55,57,59-61]$, but this is of course not an accepted standard yet. 
For resectable colorectal cancer liver metastasis, low perioperative morbidity and mortality with long-term survival comparable to hepatic resection in carefully selected patients has been reported [55]. In particular patients with hepatic metastases smaller than 3 $\mathrm{cm}$ and no tumors within $1 \mathrm{~cm}$ of central biliary structures showed a benefit in this study.

Furthermore, ablation techniques (i.e. RFA) have also been shown to be effective in controlling symptoms and to optimize quality of life in patients suffering from metastatic pancreatic neuroendocrine tumors $[62,63]$. Very few studies have specifically analyzed the outcomes of ablation techniques for PDAC liver metastasis [64]. In a retrospective study, RFA of liver metastases was performed on 34 patients with PDAC after pancreatic resection or intraoperatively at the time of resection [65]. Criteria for RFA were liver metastasis up to $3 \mathrm{~cm}$ diameter in size, five or fewer lesions, and no other distant metastases. The interval between pancreatic resection and liver metastasis was 3 months (range 0-33 months). The median survival time after liver metastasis ablation was 14 months. Another retrospective series reported the outcome of using SBRT in 27 patients with liver metastasis from unfavorable primaries including 8 pancreatic cancer patients [66]. The authors found 2-year local control and overall survival rates of 85 and 38\%, respectively, which suggests that both approaches could be feasible strategies for extending survival in selected PDAC patients with oligometastatic burden of the liver. To further evaluate this approach, future research within controlled prospective clinical trials is urgently needed.

\section{Pulmonary Metastasis}

Genetic alterations present in metastatic lesions reflect the mutational landscape in the founder clone and might determine the metastatic pattern of PDAC [67]. Isolated pulmonary metastasis in PDAC is infrequently encountered and might define a biologically distinct subgroup [68]. This observation is supported by data from our pancreatic center where the course of 40 PDAC patients with isolated pulmonary metastasis was analyzed [69]. 22 patients presented with pulmonary metastasis after initial resection of the primary whereas 5 patients had progression of locally advanced and therefore unresectable disease. Median survival after diagnosis of pulmonary metastasis was 25.5 months; however, when patients with less than 10 lung metastases were compared to the remaining patients, a significantly improved median survival of 31.3 versus 18.7 months was reported. The same was true for unilateral localization of lung involvement (31.3 vs. 21.8 months). These patients might therefore indicate distinct clinical and genetic subgroups. Intriguingly, recurrence of metastasis to the lung after initial primary tumor resection is associated with the best long-term survival of at least 5 years for any patient with metastatic PDAC [70]. Although resection of pulmonary metastasis has been shown to provide a survival benefit for colorectal cancer patients [71-75], data for PDAC on this topic is extremely limited. In a retrospective study from Johns Hopkins University analyzing 31 patients with isolated metastases, a significantly improved median overall survival of 52 versus 22 months ( $\mathrm{p}=0.04)$ was demonstrated for patients undergoing resection of metastases $(n=9)$ [68].

Additionally, there was a trend in favor of pulmonary resection for post-relapse survival. Patients undergoing resection had a median survival after recurrence of 18.6 months, compared with only 7.5 months for non-surgical patients. It is again important to note that patients in this study were highly selected and had a good biologic tumor character identified by a favorable response to systemic therapy. In addition, patients undergoing metastasectomy had a relatively long interval between initial pancreatectomy and pulmonary relapses. Another study from Japan analyzed Japanese case reports of metachronous pulmonary metastases from PDAC [76]. They found 17 case reports published between 1983 and 2014 dealing with pulmonary metastasectomy for PDAC. The median survival after pulmonary resection was 37 months, and the 3 - and 5 -year survival rates were 50 and $41 \%$, respectively. 14 patients had disease-free intervals after resection of the primary pancreatic tumor of more than 20 months. These patients had a longer median survival after lobectomy (46 vs. 25.5 months; $\mathrm{p}=0.19$ ). 7 patients had lung metastasis of less than $16 \mathrm{~mm}$. These patients also had a significantly longer overall survival after pulmonary resection (83 vs. 16 months; $\mathrm{p}=0.04$ ). Even if general considerations need to be drawn with caution, this data implies that patients with at least isolated metachronous pulmonary metastasis might benefit from surgical resection. In the recent decade, SBRT has emerged as an effective alternative resulting in local control rates exceeding $90 \%$ in mixed cohorts with very low toxicity $[77,78]$. Although data specifically addressing lung metastases from PDAC have not been published, SBRT might serve as an alternative treatment for patients in which surgery is not a suitable option.

\section{Conclusion}

Taken together, highly selected patients suffering from synchronous and metachronous oligometastatic PDAC may potentially benefit from surgical resection with an acceptable morbidity. In order to further prove or disprove the feasibility and efficacy of such an approach, a prospective multicenter trial, in which survival and quality of life after metastatic resection and systemic chemotherapy is evaluated, has to be launched. Further research is needed to determine the benefit of local and locoregional ablation techniques or SBRT as therapeutic options for isolated liver metastases in PDAC patients.

\section{Disclosure Statement}

The authors declare no competing interests. 


\section{References}

1 Rahib L, Smith BD, Aizenberg R, Rosenzweig AB, Fleshman JM, Matrisian LM: Projecting cancer incidence and deaths to 2030: the unexpected burden of thyroid, liver, and pancreas cancers in the United States. Cancer Res 2014;74:2913-2921.

2 Quante AS, Ming C, Rottmann M, et al: Projections of cancer incidence and cancer-related deaths in Germany by 2020 and 2030. Cancer Med 2016;5:26492656.

3 Siegel RL, Miller KD, Jemal A: Cancer statistics, 2016. CA Cancer J Clin 2016;66:7-30.

4 Werner J, Combs SE, Springfeld C, Hartwig W, Hackert T, Büchler MW: Advanced-stage pancreatic cancer: therapy options. Nat Rev Clin Oncol 2013;10:323-333.

5 Ryan DP, Hong TS, Bardeesy N: Pancreatic adenocarcinoma. N Engl J Med 2014;371:1039-1049.

6 Yachida S, Iacobuzio-Donahue CA: The pathology and genetics of metastatic pancreatic cancer. Arch Pathol Lab Med 2009;133:413-422.

7 Kamisawa T, Isawa T, Koike M, Tsuruta K, Okamoto A: Hematogenous metastases of pancreatic ductal carcinoma. Pancreas 1995;11:345-349.

8 Embuscado EE, Laheru D, Ricci F, et al: Immortalizing the complexity of cancer metastasis: genetic features of lethal metastatic pancreatic cancer obtained from rapid autopsy. Cancer Biol Ther 2005;4:548-554.

9 Disibio G, French SW: Metastatic patterns of cancers: results from a large autopsy study. Arch Pathol Lab Med 2008;132:931-939.

10 Mao C, Domenico DR, Kim K, Hanson DJ, Howard JM: Observations on the developmental patterns and the consequences of pancreatic exocrine adenocarcinoma. Findings of 154 autopsies. Arch Surg 1995;130: 125-134.

11 Lemke J, Scheele J, Kapapa T, Wirtz CR, Henne-Bruns D, Kornmann M: Brain metastasis in pancreatic cancer. Int J Mol Sci 2013;14:4163-4173.

12 Rao R, Sadashiv SK, Goday S, Monga D: An extremely rare case of pancreatic cancer presenting with leptomeningeal carcinomatosis and synchronous intraparenchymal brain metastasis. Gastrointest Cancer Res 2013; 6:90-92.

13 Mirrakhimov AE, Khan FN: Epidural brain metastases in a patient with early onset pancreatic cancer: a case report and literature review. Case Rep Oncol Med 2012;2012:962305

14 Kolokythas A, Miloro MB, Olsson AB, Miloro M: Metastatic pancreatic adenocarcinoma to the mandibular condyle: a rare clinical presentation. J Oral Maxillofac Surg 2014;72:83-88.

15 Monson BK, Patel BCK, Kim CH: Metastatic mucinous adenocarcinoma of the orbit. Orbit 2011;30:18 20.

16 Webber NP, Sharma S, Grossmann AH, et al: Metastatic pancreatic adenocarcinoma presenting as a large pelvic mass mimicking primary osteogenic sarcoma: a series of two patient cases. J Clin Oncol 2010;28:e545549.

17 Bellows C, Gage T, Stark M, McCarty C, Haque S: Metastatic pancreatic carcinoma presenting as colon carcinoma. South Med J 2009;102:748-750.

18 Vähätalo K, Ekfors T, Syrjänen S: Adenocarcinoma of the pancreas metastatic to the mandible. J Oral Maxillofac Surg 2000;58:110-114.

19 Rosser CJ, Gerrard E: Metastatic adenocarcinoma of the pancreas to the testicle: a case report. Am J Clin Oncol 1999;22:619-620

20 Neoptolemos JP, Stocken DD, Friess H, et al: A randomized trial of chemoradiotherapy and chemotherapy after resection of pancreatic cancer. N Engl J Med 2004 350:1200-1210.
21 Oettle H, Post S, Neuhaus P, et al: Adjuvant chemotherapy with gemcitabine vs observation in patients undergoing curative-intent resection of pancreatic cancer: a randomized controlled trial. JAMA 2007;297: 267-277.

22 Conroy T, Desseigne F, Ychou M, et al: FOLFIRINOX versus gemcitabine for metastatic pancreatic cancer. $\mathrm{N}$ Engl J Med 2011;364:1817-1825.

23 Von Hoff DD, Ervin T, Arena FP, et al: Increased survival in pancreatic cancer with nab-paclitaxel plus cemcitabine. N Engl J Med 2013;369:1691-1703.

24 Heinemann V, Haas M, Boeck S: Systemic treatment of advanced pancreatic cancer. Cancer Treat Rev 2012;38: 843-853.

25 Tol JA, Gouma DJ, Bassi C, et al: Definition of a standard lymphadenectomy in surgery for pancreatic ductal adenocarcinoma: a consensus statement by the International Study Group on Pancreatic Surgery (ISGPS). Surgery 2014;156:591-600.

26 Paiella S, Sandini M, Gianotti L, Butturini G, Salvia R, Bassi C: The prognostic impact of para-aortic lymph node metastasis in pancreatic cancer: a systematic review and meta-analysis. Eur J Surg Oncol 2016;42: 616-624.

27 Hackert T, Büchler MW, Werner J: Current state of surgical management of pancreatic cancer. Cancers 2011;3:1253-1273.

28 Sho M, Murakami Y, Motoi F, et al: Postoperative prognosis of pancreatic cancer with para-aortic lymph node metastasis: a multicenter study on 822 patients. J Gastroenterol 2015;50:694-702.

29 Shrikhande SV, Kleeff J, Reiser C, et al: Pancreatic resection for M1 pancreatic ductal adenocarcinoma. Ann Surg Oncol 2007;14:118-127.

30 Hackert T, Niesen W, Hinz U, et al: Radical surgery of oligometastatic pancreatic cancer. Eur J Surg Oncol 2016;DOI: 10.1016/j.ejso.2016.10.023.

31 Primrose JN: Treatment of colorectal metastases: surgery, cryotherapy, or radiofrequency ablation. Gut 2002;50:1-5.

32 Weitz J, Koch M, Debus J, Höhler T, Galle PR, Büchler MW: Colorectal cancer. Lancet 2005;365:153-165.

33 Veereman G, Robays J, Verleye L, et al: Pooled analysis of the surgical treatment for colorectal cancer liver metastases. Crit Rev Oncol Hematol 2015;94:122-135.

34 Page AJ, Weiss MJ, Pawlik TM: Surgical management of noncolorectal cancer liver metastases. Cancer 2014; 120:3111-3121.

35 Schiergens TS, Lüning J, Renz BW, et al: Liver resection for non-colorectal non-neuroendocrine metastases: where do we stand today compared to colorectal cancer? J Gastrointest Surg 2016;20:1163-1172.

36 Seufferlein T, Porzner M, Becker T, et al: S3-guideline exocrine pancreatic cancer (Article in German). Z Gastroenterol 2013;51:1395-1440.

37 Tempero MA, Malafa MP, Behrman SW, et al: Pancreatic adenocarcinoma, version 2.2014: featured updates to the NCCN guidelines. J Natl Compr Canc Netw 2014;12:1083-1093.

38 Michalski CW, Erkan M, Hüser N, et al: Resection of primary pancreatic cancer and liver metastasis: a systematic review. Dig Surg 2008;25:473-480.

39 Dünschede F, Will L, von Langsdorf C, et al: Treatment of metachronous and simultaneous liver metastases of pancreatic cancer. Eur Surg Res 2010;44:209213.

40 Klempnauer J, Ridder GJ, Piso P, Pichlmayr R: Ist die Leberresektion bei Metastasen eines exokrinen Pankreascarcinoms gerechtfertigt? Chirurg 1996;67:366-370.
41 Shimada K, Kosuge T, Yamamoto J, Yamasaki S, Sakamoto M: Successful outcome after resection of pancreatic cancer with a solitary hepatic metastasis. Hepatogastroenterology 2004;51:603-605.

42 Gleisner AL, Assumpcao L, Cameron JL, et al: Is resection of periampullary or pancreatic adenocarcinoma with synchronous hepatic metastasis justified? Cancer 2007;110:2484-2492.

43 Tachezy M, Gebauer F, Janot M, et al: Synchronous resections of hepatic oligometastatic pancreatic cancer: disputing a principle in a time of safe pancreatic operations in a retrospective multicenter analysis. Surgery 2016;160:136-144.

44 Klein F, Puhl G, Guckelberger O, et al: The impact of simultaneous liver resection for occult liver metastases of pancreatic adenocarcinoma. Gastroenterol Res Pract 2012;2012:939350.

45 Yamada H, Hirano S, Tanaka E, Shichinohe T, Kondo S: Surgical treatment of liver metastases from pancreatic cancer. HPB (Oxford) 2006;8:85-88.

46 Klempnauer J, Ridder GJ, Bektas H, Pichlmayr R: Extended resections of ductal pancreatic cancer - impact on operative risk and prognosis. Oncology 1996;53: 47-53.

47 Takada T, Yasuda H, Amano H, Yoshida M, Uchida T: Simultaneous hepatic resection with pancreato-duodenectomy for metastatic pancreatic head carcinoma: does it improve survival? Hepatogastroenterology 1997; 44:567-573.

48 Seelig SK, Burkert B, Chromik AM, Tannapfel A, Uhl W, Seelig MH: Pancreatic resections for advanced M1pancreatic carcinoma: the value of synchronous metastasectomy. HPB Surg 2010;2010:579672.

49 Hartwig W, Strobel O, Hinz U, et al: CA19-9 in potentially resectable pancreatic cancer: perspective to adjust surgical and perioperative therapy. Ann Surg Oncol 2013;20:2188-2196.

50 de Baere T, Deschamps F: Treatment of hepatic and pulmonary metastases with radiofrequency. Diagn Interv Imaging 2014;95:672-677.

51 de Baere T, Deschamps F: New tumor ablation techniques for cancer treatment (microwave, electroporation). Diagn Interv Imaging 2014;95:665-671.

52 Chen J, Tang Z, Dong X, et al: Radiofrequency ablation for liver metastasis from gastric cancer. Eur J Surg Oncol 2013;39:701-706

53 Viganò L, Russolillo N, Ferrero A, et al: Resection of liver metastases from colorectal mucinous adenocarcinoma: is this a different disease? Results of a casecontrol study. Ann Surg 2014;260:878-884; discussion 884-885.

54 Sofocleous CT, Sideras P, Petre EN: 'How we do it' - a practical approach to hepatic metastases ablation techniques. Tech Vasc Interv Radiol 2013;16:219-229.

55 Simo KA, Sereika SE, Newton KN, Gerber DA: Laparoscopic-assisted microwave ablation for hepatocellular carcinoma: safety and efficacy in comparison with radiofrequency ablation. J Surg Oncol 2011;104:822829.

56 Ahmed KA, Torres-Roca JF: Stereotactic body radiotherapy in the management of oligometastatic disease. Cancer Control 2016;23:21-29.

57 Scorsetti M, Comito T, Tozzi A, et al: Final results of a phase II trial for stereotactic body radiation therapy for patients with inoperable liver metastases from colorectal cancer. J Cancer Res Clin Oncol 2014;141:543-553.

58 Gillams A, Goldberg N, Ahmed M, et al: Thermal ablation of colorectal liver metastases: a position paper by an international panel of ablation experts, The Interventional Oncology Sans Frontières meeting 2013. Eur Radiol 2015;25:3438-3454. 
59 Zimmermann A, Geiger R, Kortmann H: Similarity between a kininogenase (kallikrein) from human large intestine and human urinary kallikrein. Hoppe Seylers Z Physiol Chem 1979;360:1767-1773.

60 Hammill CW, Billingsley KG, Cassera MA, Wolf RF, Ujiki MB, Hansen PD: Outcome after laparoscopic radiofrequency ablation of technically resectable colorectal liver metastases. Ann Surg Oncol 2011;18:1947-1954.

61 Ripley RT, Kemp CD, Davis JL, et al: Liver resection and ablation for metastatic adrenocortical carcinoma. Ann Surg Oncol 2011;18:1972-1979.

62 O'Grady HL, Conlon KC: Pancreatic neuroendocrine tumours. Eur J Surg Oncol 2008;34:324-332.

63 Moug SJ, Leen E, Horgan PG, Imrie CW: Radiofrequency ablation has a valuable therapeutic role in metastatic VIPoma. Pancreatology 2006;6:155-159.

64 De Jong MC, Farnell MB, Sclabas G, et al: Liver-directed therapy for hepatic metastases in patients undergoing pancreaticoduodenectomy: a dual-center analysis. Ann Surg 2010;252:142-148.

65 Park JB, Kim YH, Kim J, et al: Radiofrequency ablation of liver metastasis in patients with locally controlled pancreatic ductal adenocarcinoma. J Vasc Interv Radiol 2012;23:635-641.
66 Yuan Z-Y, Meng M-B, Liu C-L, et al: Stereotactic body radiation therapy using the CyberKnife ${ }^{\circledR}$ system for patients with liver metastases. Onco Targets Ther 2014;7: 915-923.

67 Yachida S, Jones S, Bozic I, et al: Distant metastasis occurs late during the genetic evolution of pancreatic cancer. Nature 2010;467:1114-1117.

68 Arnaoutakis GJ, Rangachari D, Laheru DA, et al: Pulmonary resection for isolated pancreatic adenocarcinoma metastasis: an analysis of outcomes and survival. J Gastrointest Surg 2011;15:1611-1617.

69 Kruger S, Haas M, Burger PJ, et al: Isolated pulmonary metastases define a favorable subgroup in metastatic pancreatic cancer. Pancreatology 2016;16:593-598.

70 Katz MHG, Wang H, Fleming JB, et al: Long-term survival after multidisciplinary management of resected pancreatic adenocarcinoma. Ann Surg Oncol 2009;16: 836-847.

71 Goya T, Miyazawa N, Kondo H, Tsuchiya R, Naruke T, Suemasu K: Surgical resection of pulmonary metastases from colorectal cancer. 10-year follow-up. Cancer 1989;64:1418-1421.

72 Pfannschmidt J, Dienemann H, Hoffmann H: Surgical resection of pulmonary metastases from colorectal cancer: a systematic review of published series. Ann Thorac Surg 2007;84:324-338.
73 Limmer S, Oevermann E, Killaitis C, Kujath P, Hoffmann M, Bruch H-P: Sequential surgical resection of hepatic and pulmonary metastases from colorectal cancer. Langenbecks Arch Surg 2010;395:1129-1138.

74 Sakamoto Y, Sakaguchi Y, Oki E, Minami K, Toh Y, Okamura T: Surgical outcomes after resection of both hepatic and pulmonary metastases from colorectal cancer. World J Surg 2012;36:2708-2713.

75 Suzuki H, Kiyoshima M, Kitahara M, Asato Y, Amemiya R: Long-term outcomes after surgical resection of pulmonary metastases from colorectal cancer. Ann Thorac Surg 2015;99:435-440.

76 Nakajima M, Ueno T, Suzuki N, et al: Novel indications for surgical resection of metachronous lung metastases from pancreatic cancer after curative resection. J Clin Gastroenterol 2016;DOI: 10.1097/MCG.0000000000000551.

77 Okunieff P, Petersen AL, Philip A, et al: Stereotactic body radiation therapy (SBRT) for lung metastases. Acta Oncol 2009;45:808-817.

78 Rusthoven KE, Kavanagh BD, Burri SH, et al: Multiinstitutional phase I/II trial of stereotactic body radiation therapy for lung metastases. J Clin Onc 2009;27: 1579-1584. 